\title{
Estructuras y mecanismos mentales de los estudiantes en los conceptos de permutación y combinación analizadas desde la teoría apõe
}

\section{Students' mental structures and mechanisms in the concepts of permutation and combination analyzed from the apoe theory}

DOI: $10.46932 / \mathrm{sfjdv} 3 \mathrm{n} 1-001$

Received in: Dec 30st, 2021

Accepted in: Jan 1th, 2022

\author{
Joaquin Ignacio Cartes Veliz \\ Pedagogía en Matemática, Universidad de Concepción (2021) \\ Liceo Bicentenario Técnico Profesional Alonso de Ercilla y Zúñiga \\ Río Cayucupil 087, Cañete, Chile \\ Correo: joaquin.cartesveliz@educanete.cl \\ Juan Patricio Soto Díaz \\ Pedagogía en Matemática, Universidad de Concepción (2021) \\ Liceo Pencopolitano \\ Longitudinal Dos 945, San Pedro de la Paz, Chile \\ Correo: juan.soto@liceopencopolitano.cl \\ José Manuel Valencia Bravo \\ Pedagogía en Matemática, Universidad de Concepción (2021) \\ Universidad de Concepción \\ Dos oriente 224, Dpto 211, Talca, Chile \\ Correo: jvalencia2016@udec.cl
}

\begin{abstract}
RESUMEN
Este trabajo presenta una investigación en el área de la didáctica de la matemática fundamentada mediante la teoría APOE (Acrónimo de Acción, Proceso, Objeto y Esquema) formalizada en Asiala et al. (1996), la que permite analizar la forma en que los estudiantes construyen mentalemente cualquier concepto matemático, clasificando dichas construcciones en estructuras y mecanismos mentales. Se trabajó específicamente con los conceptos de permutación y combinación que se encuentran presentes en el nivel de segundo medio del plan de estudio de matemática y son fundamentales para el cálculo de probabilidades. Para efectos de este análisis se presenta una hipótesis sobre cómo los estudiantes aprenden los conceptos, denominada "Descomposición Genética" (DG), la cual se refina mediante la aplicación de cuestionarios y entrevistas a estudiantes de diferentes niveles de enseñanza media y profesores de matemática en formación para determinar cómo construyen los conceptos de interés y sugerir a los profesores una secuencia de construcción de los conceptos de permutación y combinación por medio de la resolución de problemas.
\end{abstract}

Palabras claves: Teoría APOE, Estructuras y mecanismos mentales, Descomposición Genética, Permutación, Combinación.

\section{ABSTRACT}

This paper presents an investigation in the area of mathematics didactics based on the APOS Theory (Acronym for Action, Process, Object and Scheme) formalized in Asiala et al. (1996), which allows 
analyzing the way in which students mentally construct any mathematical concept, classifying these constructions in mental structures and mechanisms. We worked specifically with the concepts of permutation and combination, which are present in the second level of the mathematics curriculum and are fundamental for the calculation of probabilities. For the purposes of this analysis, a hypothesis is presented on how students learn the concepts, called "Genetic Decomposition" (GD), which is refined through the application of questionnaires and interviews with students from different levels of secondary education and mathematics teachers in training to determine how they construct the concepts of interest and to suggest to teachers a sequence of construction of the concepts of permutation and combination by means of problem solving.

Keywords: APOS Theory, Mental Structures and Mechanisms, Genetic Decomposition, Permutation, Combination.

\section{INTRODUCCIÓN}

El área que se trabajó en esta investigación es la combinatoria, específicamente los conceptos de permutación y combinación, insertos en el programa de estudios de segundo medio en el eje temático Probabilidad y estadística como herramienta para el cálculo de probabilidades. Dichos conceptos son fundamentales para los estudiantes de dicho nivel en su escolaridad, los cuales presentan dificultades en su enseñanza y aprendizaje, tal como se manifiesta en Salgado (2008): "Los problemas de conteo se introducen en la escuela desde la enseñanza primaria y tanto profesores como alumnos muestran dificultades con los conceptos involucrados. Estos problemas se siguen manifestando en el nivel universitario".

Para analizar la forma en que los estudiantes construyen los conceptos de interés se utilizó el ciclo de investigación propuesto por la teoría APOE y utilizada por el grupo RUMEC (Research in Undergraduate Mathematics Education Community), el cual contempla tres fases. En primer lugar, se presenta el análisis previo que se realizó (concepto-epistemología-currículum), seguido de la construcción de la descomposición genética (DG) e instrumento, para finalizar con el análisis de las respuestas y la aplicación de entrevistas. Las fases anteriores permiten realizar un refinamiento a la DG.

La investigación busca aportar al proceso de enseñanza-aprendizaje de los estudiantes al encontrar una forma de abordar de manera conceptual las combinaciones y permutaciones, a partir de esquemas mentales que construyen los mismos estudiantes al momento de resolver problemas en esta área, basados en la teoría APOE, por lo que nuestra pregunta de investigación es: ¿Qué estructuras y mecanismos mentales utilizan los estudiantes de diferentes establecimientos de la región del Biobío cuando resuelven problemas donde están involucrados los conceptos de permutación y combinación de acuerdo a su nivel de estudios? 


\section{ANTECEDENTES}

Para la realización del trabajo se recabaron antecedentes de tipo epistemológico (desarrollo histórico de los conceptos), conceptual (conceptualización matemática formal), curricular (presencia de los conceptos en el currículum de matemática escolar) e investigaciones ya existentes que abordan la temática de interés.

El análisis epistemológico basado en Biggs (1979) y Domínguez (2016) de los conceptos de permutación y combinación, permite evidenciar que estos fueron abordados a lo largo de la historia en primer lugar de manera intuitiva, como simples problemas o acertijos que podían ser resueltos sin un mayor bagaje matemático desde aproximadamente 2000 años a.C. en China y posteriormente en varias civilizaciones del mundo como India o Grecia. Dichos conceptos pasaron desapercibidos por los matemáticos a lo largo de la historia, generando interés cerca de 1650 en francia con la implementación de los juegos de azar y recién se llegaron a formalizar alrededor del año 1666 con la publicación del libro "De arte Combinatoria" de Leibniz y el envío de una carta del mismo a Bernoulli en 1699 que permite que nazca la teoría de las particiones. De estos avances que se presentan como ejemplo surge la combinatoria como disciplina matemática.

En cuanto al análisis conceptual matemático, podemos notar que en Grimaldi (1998) se precisa una definición de los conceptos de permutación y combinación utilizando como base la regla del producto (principio multiplicativo).

Para efectos del análisis curricular, se realizó una revisión del plan de estudios de matemática en todos los niveles escolares para identificar en dónde estaban presentes los conceptos de interés, los niveles previos y los niveles posteriores. Los conceptos de interés se encuentran presentes en el nivel de segundo medio y tienen por objetivo utilizar permutaciones y combinaciones para el cálculo de probabilidades. En los niveles previos destacan los niveles de séptimo básico, octavo básico y primero medio en donde se emplea el principio multiplicativo para obtener el total de posibilidades en un determinado evento y en los niveles posteriores se encuentran en tercero y cuarto medio (plan común y electivos) el uso de permutaciones para la construcción de la distribución binomial de probabilidad.

Algunas investigaciones fundamentadas mediante la teoría APOE que abordan la temática de interés y que apoyan este trabajo son Salgado y Trigueros (2009) y Domínguez (2016) en dónde se presenta una DG de los conceptos de interés para nivel universitario y secundaria respectivamente, con el objetivo de construir una propuesta didáctica en el área.

\section{METODOLOGÍA}

La investigación es cualitativa y usa la metodología del ciclo de investigación de APOE trabajada por el grupo RUMEC (Research in Undergraduate Mathematics Education Community), y que establece 
tres fases: i. Análisis teórico del concepto, ii. Diseño e implementación de enseñanza y iii. Observación, análisis y verificación de datos.

Así, como primera acción se realiza un análisis epistemológico, curricular y matemático de los conceptos de permutación y combinación, constituyendo hipótesis sobre cómo los estudiantes aprenden los conceptos ya mencionados, estableciendo la "Descomposición Genética preliminar" la cual se encuentra categorizada en tablas de estructuras y mecanismos mentales y que en resumen, considera que los estudiantes construyen los conceptos siguiendo una secuencia, primeramente de manera intuitiva y posteriormente generalizando, o bien, aplican los conceptos ya conocidos. La secuencia a nivel de contenido es principio multiplicativo-permutación-combinación.

En la segunda fase, se diseña un instrumento con tres problemas considerando la descomposición genética desarrollada, que abordan los conceptos de principio multiplicativo, permutación y combinación respectivamente, aplicados a estudiantes en dos oportunidades (primer y segundo testeo), categorizando las respuestas en base a la DG en la tercera fase. En esta última también se realizan entrevistas para observar mecanismos mentales y así poder refinar la DG preliminar, obteniendo conclusiones sobre cómo los estudiantes aprenden los conceptos de permutación y combinación.

\section{ANÁLISIS}

Se realizó un primer y segundo testeo del instrumento a estudiantes de enseñanza media y profesores de matemática en formación, incluyendo entrevistas para obtener mayor información de los mecanismos mentales. El primer testeo arrojó información de estudiantes que respondían correctamente validando la DG, y otros que no respondían a lo solicitado debido a la poca comprensión de los enunciados. Es por esto último que se decide mejorar el instrumento en cuanto a su redacción, considerando las respuestas de los estudiantes, para ser implementado en un segundo testeo (sin modificar la DG preliminar) con el fin de obtener datos más recabados.

El análisis (en el primer y segundo testeo) fue realizado en base a las respuestas de los estudiantes, se toma la imagen de la respuesta entregada por el estudiante, considerando tanto errores como aciertos, y cada una de las producciones que realiza se clasifican en base a las etapas de la teoría APOE (Acción, Proceso, Objeto, Esquema) justificando el por qué manifiesta estar en esa etapa, además, se buscan indicios que indiquen el uso de mecanismos mentales, pero estos son abordados con mayor precisión por medio de entrevistas.

A modo de ejemplo, se presenta la respuesta de un estudiante a la situación 1: "Camila a la Moda", la cual contempla cuatro incisos y consiste en encontrar el total de combinaciones posibles de vestimenta con una determinada cantidad de prendas distintas (poleras, pantalones, etc.). Cada inciso va añadiendo una determinada cantidad de prendas hasta llegar al último, que demanda determinar el total de 
combinaciones de vestimenta con una cantidad general de prendas de cada tipo (a cantidad de prenda 1, b cantidad de prenda $2, \ldots$, g cantidad de prenda 7 ).

Figura 1. Respuesta estudiante 2, Situación 1.d. - Segundo testeo

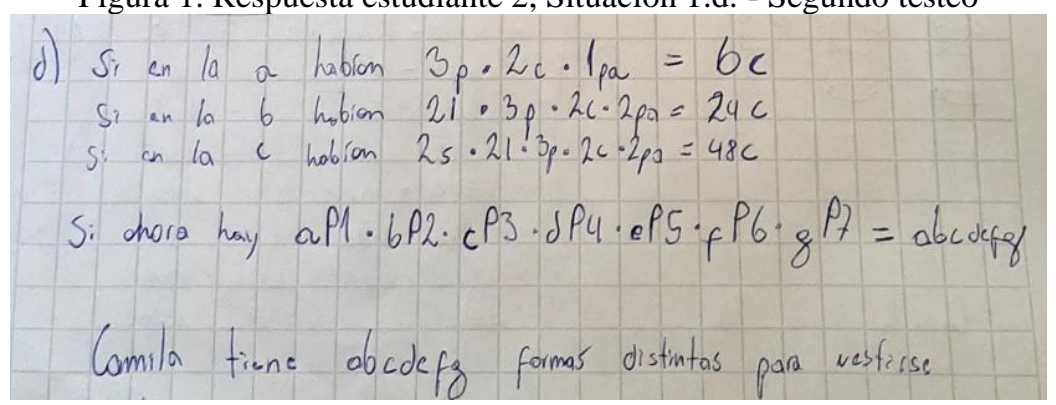

Fuente: Elaboración estudiante 2, segundo medio

De la respuesta anterior, se puede observar que el estudiante utiliza los resultados obtenidos en los incisos anteriores y los relaciona con la cantidad de prendas a modo de multiplicación, por ejemplo en el inciso a indica que habían 3 pantalones (p), 2 cinturones (c) y 1 polera (po) que al multiplicar resulta 6 combinaciones (c). Lo anterior se encuentra en etapa de proceso según la DG. Al repetir este proceso en cada uno de los incisos el estudiante logra encontrar una forma de escribir todas las combinaciones posibles en el inciso d obteniendo la respuesta correcta y logrando un acercamiento a la construcción de la fórmula del principio multiplicativo, lo cual se encuentra en etapa de objeto según nuestra DG.

Por otro lado, se realiza una entrevista al estudiante sobre esta misma respuesta para indagar en el uso de mecanismo, el estudiante nos afirma:

\begin{abstract}
“(...) con la pequeña teoría que se me ocurrió de multiplicarlo todo a ver si me daba. En la b) lo mismo: 2 lentes, 3 poleras, 2 cinturones, 2 pantalones: 24. Y así lo mismo en la c). En la d), se decía que se multiplicaba ap1, bp2, cp3, dp4, ep5, fp6, gp7. Es lo que dije. Si le saco todas las letras y solo multiplico los números, en las otras, me iba a dar el total de combinaciones. Entonces, hice lo mismo, pero, sólo saqué los p1, p2, p3 y multipliqué solo las letras. Que me dió de que Camila tenía abcdefg formas distintas para vestirse". (Estudiante 2, comunicación personal, 11 de noviembre de 2020).
\end{abstract}

Consideramos que el estudiante está utilizando el mecanismo de "coordinación” pues extrae elementos importantes de cada uno de los procesos que realizó en los incisos anteriores dando una conclusión (le dice pequeña teoría) y que es el multiplicar cada una de las prendas. Por medio de esto, creemos que el estudiante es capaz de llegar a una "encapsulación" construyendo el objeto de principio multiplicativo, siendo capaz de escribir todas las combinaciones de prendas distintas de manera general como abcdefg formas distintas. 


\section{CONCLUSIONES}

Las conclusiones se clasifican en tres categorías: a) estructuras y mecanismos nuevos (o modificados) que permiten refinar la DG preliminar; b) sugerencias de mejora a los tres problemas implementados; c) conclusiones respecto a la enseñanza y aprendizaje de los conceptos.

a) La DG preliminar fue validada en su mayoría a partir de las respuestas de los estudiantes, surgiendo nuevas estructuras y mecanismos que permiten refinarla. Como por ejemplo, que los estudiantes realicen listas completas de combinaciones o reordenamientos posibles para obtener el total (acción), ya habiendo pasado por el principio multiplicativo, lo que se creía que no ocurriría producto de la generalización previa (encapsulación del objeto).

b) Los problemas iniciales requieren ser modificados de manera que permitan que los estudiantes los resuelvan mediante diversos procedimientos domine o no los conceptos involucrados. Cada situación debe contener una serie de preguntas (incisos) secuenciadas desde lo particular a lo general.

c) Se sugiere implementar la secuencia de trabajo principio multiplicativo- permutación-combinación en el aula considerando las sugerencias expuestas, utilizando problemas que permitan el uso de diversos procedimientos para maximizar los mecanismos que permiten un tránsito entre diversas estructuras mentales, con el objetivo de lograr la encapsulación de los conceptos, fundamentales en el área de la combinatoria y el cálculo de probabilidades. 


\section{BIBLIOGRAFÍA}

Asiala, M., Brown, A., DeVries, D., Dubinsky, E., Mathews, D. \& Thomas, K. (1996). A Framework for Research and Curriculum Development in Undergraduate Mathematics Education. In J. Kaput, A. H. Schoenfeld \& E. Dubinsky (Eds.), Research in Collegiate Mathematics Education II (pp.1-32). U.S.A.: American Mathematical Society

Biggs, N. L. (1979, mayo). The roots of combinatorics. Historia Matemática, 6(2), 109-136. https://www.sciencedirect.com/science/article/pii/0315086079900740\#aep-bibliography-id7

Domínguez, D. L. (2016). Secuencia didáctica que le permite a los estudiantes de octavo y noveno interpretar y usar las nociones de conteo en la solución de problemas de combinación y permutación. Maestría thesis, Universidad Nacional de Colombia-SedeBogotá. http://bdigital.unal.edu.co/54569/7/DianaLuc\%C3\%ADaDominguezP.2016.pdf

Grimaldi, R. (1998). Matemáticas Discreta y Combinatoria: Una introducción con aplicaciones, Naucalpan de Juárez, México. Addison Wesley Iberoamericana S.A.

Ministerio de educación (s.f.) Matemática $2^{\circ}$ medio Unidad 4: Probabilidades. https://www.curriculumnacional.cl/portal/Educacion-General/Matematica/Matematica-2medio/79943:Unidad-4-Probabilidades

Salgado, H. \& Trigueros, M. (2009). Conteo: una propuesta didáctica y su análisis. Educación matemática, 21(1), 91-117. http://www.scielo.org.mx/scielo.php?script=sci_arttext\&pid=S1665$58262009000100005 \& \operatorname{lng}=$ es\&tng=es. 